\title{
Uncertainty quantification of elastic material responses: testing, stochastic calibration and Bayesian model selection
}

\author{
Danielle Fitt ${ }^{1} \cdot$ Hayley Wyatt ${ }^{2} \cdot$ Thomas E. Woolley $^{1} \cdot$ L. Angela Mihai ${ }^{2}$ (i) \\ Received: 3 September 2019 / Accepted: 9 October 2019 / Published online: 23 November 2019 \\ (C) The Author(s) 2019
}

\begin{abstract}
Motivated by the need to quantify uncertainties in the mechanical behaviour of solid materials, we perform simple uniaxial tensile tests on a manufactured rubber-like material that provide critical information regarding the variability in the constitutive responses between different specimens. Based on the experimental data, we construct stochastic homogeneous hyperelastic models where the parameters are described by spatially independent probability density functions at a macroscopic level. As more than one parametrised model is capable of capturing the observed material behaviour, we apply Bayes' theorem to select the model that is most likely to reproduce the data. Our analysis is fully tractable mathematically and builds directly on knowledge from deterministic finite elasticity. The proposed stochastic calibration and Bayesian model selection are generally applicable to more complex tests and materials.
\end{abstract}

Keywords Stochastic elasticity · Finite strain analysis · Hyperelastic material · Bayes' factor · Experiments $\cdot$ Probabilities

"This task is made more difficult than it otherwise would be by the fact that some of the test-pieces used have to be moulded individually, and it is difficult to make two rubber specimens having identical properties even if nominally identical procedures are followed in preparing them." - R.S. Rivlin and D.W. Saunders [55]

\section{Introduction}

The study of material elastic properties has traditionally used deterministic approaches, based on ensemble averages, to quantify constitutive parameters [36]. In practice, these parameters can meaningfully take on different values corresponding to possible outcomes of the experiments. The art and challenge of experimental setup is to get as close as possible to the ideal situations that can be analysed mathematically. From the mathematical modelling point of view, stochastic representations accounting for data dispersion are needed to improve assessment and predictions $[9,14,18,26,45,50,61,71]$.

Recently, stochastic models (described by a strain-energy density) were proposed for nonlinear elastic materials, where the parameters are characterised by probability distributions at a continuum level [37, 65-69]. These are advanced

L. Angela Mihai

MihaiLA@ cardiff.ac.uk

Danielle Fitt

FittD@cardiff.ac.uk

Hayley Wyatt

WyattHL@cardiff.ac.uk

Thomas E. Woolley

WoolleyT1@ cardiff.ac.uk

1 School of Mathematics, Cardiff University, Senghennydd Road, Cardiff, CF24 4AG, UK

2 School of Engineering, Cardiff University, The Parade, Cardiff, CF24 3AA, UK 
phenomenological models that rely on the finite elasticity theory $[15,48,76]$ and on the maximum entropy principle to enable the propagation of uncertainties from input data to output quantities [62]. The principle of maximum entropy was introduced by Jaynes (1957) [19, 20] (see also [21]) and states that "The probability distribution which best represents the current state of knowledge is the one with largest entropy (or uncertainty) in the context of precisely stated prior data (or testable information)." The notion of entropy (or uncertainty) of a probability distribution was first defined by Shannon (1948) [57, 64] in the context of information theory. These models can further be incorporated into Bayesian approaches [3, 30] for model selection and updates [37, 45, 46, 56].

For non-deterministic material models, two important questions arise, namely "how do material constitutive laws influence possible equilibrium states and their stability?" and "what is the effect of the probabilistic parameters on the predicted elastic responses?" Presently, theoretical approaches have been able to successfully contend with cases of simple geometry such as cubes, spheres, shells and tubes. Specifically, within the stochastic framework, the classic problem of the Rivlin cube was considered in [38], the static and dynamic inflation of cylindrical and spherical shells was treated in [34, 35], the static and dynamic cavitation of a sphere was analysed in [33,40], and the stretch and twist of anisotropic cylindrical tubes was examined in [39]. These problems were drawn from the analytical finite elasticity literature and incorporate random variables as basic concepts along with mechanical stresses and strains. Such problems are interesting in their own right and may also inspire further thinking about how stochastic extensions can be formulated before they can be applied to more complex systems.

To investigate the effect of probabilistic parameters in the case of more realistic geometries and loading conditions, computational approaches have been proposed in $[68,69]$. However, for real materials, most available data consist of mean values from which deterministic models are usually derived, and there is a lack of experimental data reported in the literature that are directly suitable for stochastic modelling.

For rubber-like materials, the first experimental data for load-deformation responses of different material samples under large strains were provided by Rivlin and Saunders [55]. Following the phenomenological models for vulcanised rubber developed by Treloar (2005) [75] (read also [13]), many deterministic hyperelastic models calibrated to the mean data values were described (for example, in $[7,8,17,36,49,70,77]$ ). To capture the variability of Rivlin and Saunders' data (see also [75, p. 224], or [76, p. 181]), probability distributions for the random shear modulus under relatively small strains were obtained in [34]. In recognition of the fact that a crucial part in assessing the elasticity of materials is to quantify the uncertainties in their mechanical responses, for rubber and soft tissues under large strain deformations, explicit stochastic hyperelastic models based on datasets consisting of mean values and standard deviations were developed in [37], while statistical models derived from numerically generated data were proposed in $[6,44]$. In all these developments, the aim was to find the most appropriate general method of characterising the properties of nonlinear elastic materials at a macroscopic (non-molecular) level.

In this study, we first report on simple experimental results for different samples of silicone rubber material in finite uniaxial tension (Section 2). Full details of the manufactured material are presented in Section 2.1, while specific descriptions of the experimental setup and techniques used during testing are outlined in Sections 2.2 and 2.3. Employing the stochastic calibration method and Bayesian selection criterion proposed in [37], we then obtain stochastic homogeneous hyperelastic models, where the parameters are random variables that are constant in space, and are calibrated to our experimental measurements (Section 3). The assumptions and ideas that we adopt for the stochastic modelling are discussed in Section 3.1, while calibration results and details on how Bayes' theorem can be employed explicitly to select the best performing model are given in Sections 3.3 and 3.4. In Section 4, we draw concluding remarks.

\section{Experimental testing}

In this section, we describe the experimental setup and techniques used to measure large elastic deformations of manufactured silicone rubber specimens. Our experimental results capture the inherent variability in the acquired data between different specimens during tensile tests.

\subsection{Specimen manufacture}

Two batches of silicone were manufactured in order to investigate the consistency of the specimen behaviour within the same batch and within different batches. Each sample was a simple rectangular shape with rounded edges. The approximate 


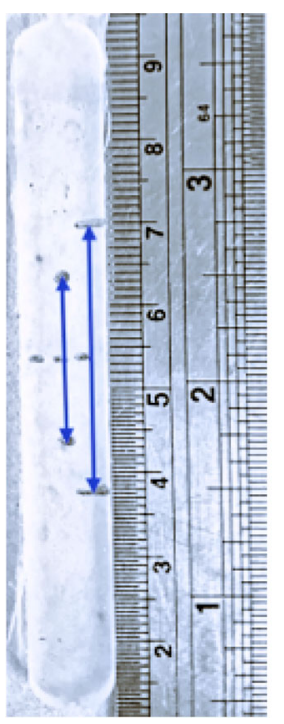

(a)

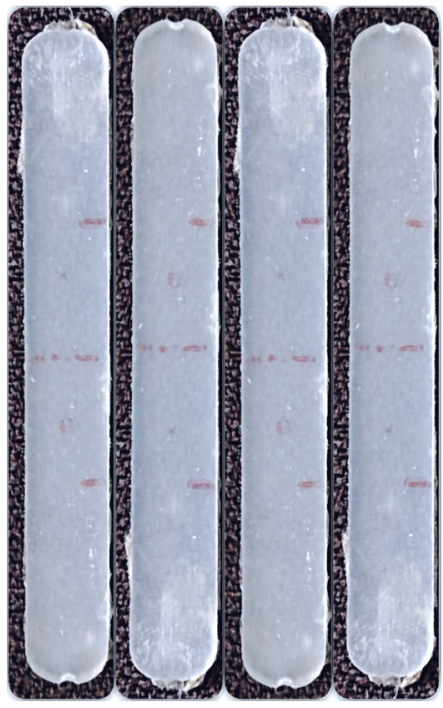

(b)

Fig. 1 Marked undeformed specimens, with height a of $100 \mathrm{~mm}$, width of $10 \mathrm{~mm}$, and depth of $4 \mathrm{~mm}$ : a single specimen where the longer double arrow shows the grip length, which was $30 \mathrm{~mm}$ as standard and reduced to $20 \mathrm{~mm}$ for some tests, while the shorter arrow indicates the gauge length, which was $20 \mathrm{~mm}$ as standard and $10 \mathrm{~mm}$ when using the reduced grip length; $\mathbf{b}$ multiple specimens

geometric parameters of each sample before deformation were height of $100 \mathrm{~mm}$, width of $10 \mathrm{~mm}$, and a depth $4 \mathrm{~mm}$. Examples of undeformed samples are illustrated in Fig. 1.

- For Batch 1, tensile testing specimens were cast using Tech-Sil 25 Silicone (Technovent). This is a two part silicone, with a standard mixture ratio of 9:1 for Part A:Part B, respectively, as per the manufacturer's recommendation, and is generally allowed to cure at room temperature for at least $24 \mathrm{~h}$. The silicone was mixed and de-gassed prior to casting, ensuring an even mixture, and no air bubbles were present in the tensile specimens. Testing specimens of equal dimensions were made within a mould that is typically used for this purpose. The silicone was removed from the mould for testing after 4 weeks.

- For Batch 2, the same make of silicone as in Batch 1 was used, with slight variations in the mixture components in order to simulate an error that would be within a realistic experimental range. Namely, the mixture ratio was 8.96:1. Tensile testing specimens with the specified geometry were created using this mixture. The silicone was also left to cure at room temperature, and taken out of the mould for testing after 2 weeks.

The full details of the silicone mixture for the two batches are recorded in Table 1.

\subsection{Experimental set-up}

Two testing sessions were conducted, to allow for variability in the experimental results due to degradation of silicone properties over different testing days. Uniaxial tests were conducted on the Zwick Roel Z050 testing machine, with a $1 \mathrm{KN}$ load cell to measure tensile force. Specimens were mounted using a set of roll clamps, and the general experiment setup can be observed in Fig. 2. The standard test method used consisted of a pre-load of $2 \mathrm{~N}$, a 30-mm grip length of the specimens,

Table 1 Full details of the silicone mixture for the two batches of tensile testing specimens

\begin{tabular}{lll}
\hline Batch number & 1 & 2 \\
\hline Part A weight $(\mathrm{g})$ & 180 & 92.3 \\
Part B weight $(\mathrm{g})$ & 20 & 10.3 \\
Mixing ratio & $9: 1$ & $8.96: 1$ \\
Curing period & 4 weeks & 2 weeks \\
Number of specimens tested & 6 & 2 \\
\hline
\end{tabular}




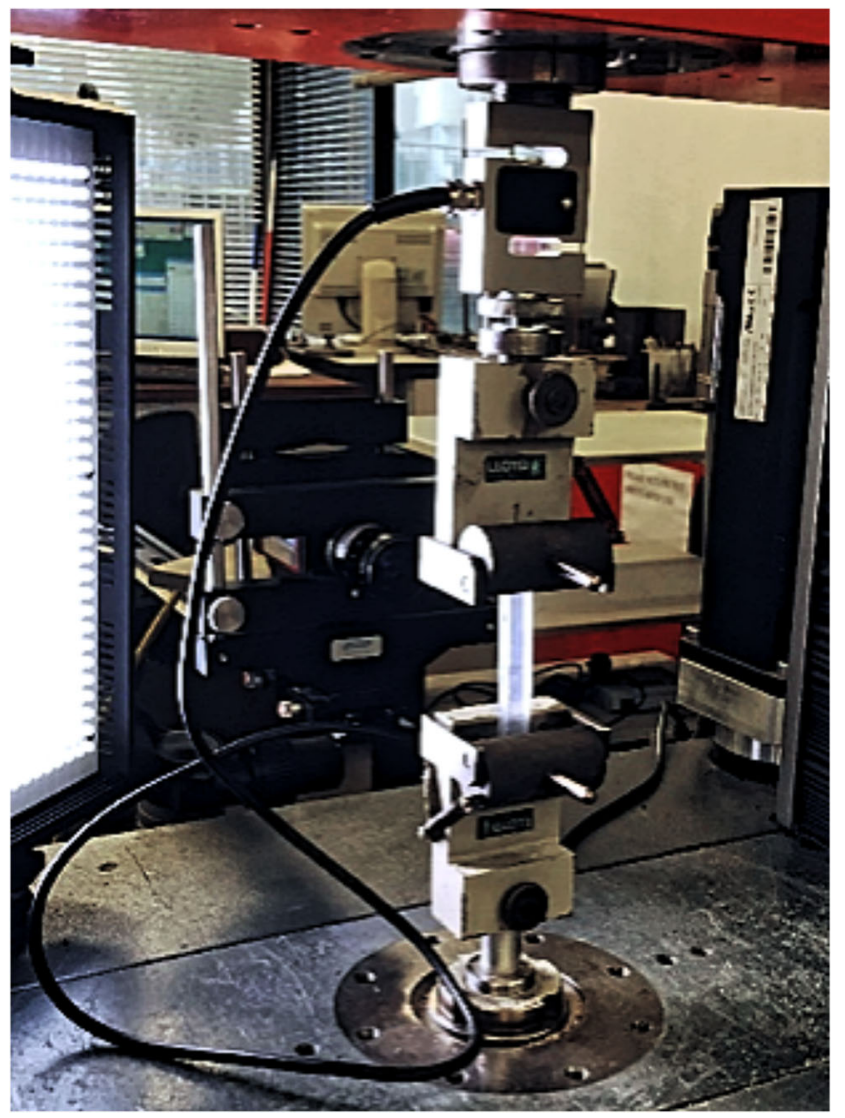

Fig. 2 Experimental setup for uniaxial tensile testing of silicone specimens mounted in roll grips, with lights and cameras for optical strain measurement

and loading at a speed of $30 \mathrm{~mm} / \mathrm{min}$. However, testing parameters, such as the grip length and testing speed, were varied between specimens, as detailed in Table 2 . Tests were stopped once specimens reached approximately 100\% strain measured optically (see Section 2.3 for details). All specimens were tested with the same technician loading the test specimens and using the test machine.

\subsection{Optical strain measurement}

A video strain gauge system (Imetrum) was employed to capture the global deformation of the specimen during tensile tests. The system works by imaging the specimen in an unloaded state, and then tracking the position of markers on the surface of the specimen throughout loading to measure displacements $[10,72,78,79]$. The system was used with a single camera with a general purpose lens, and calibrated using markers of a known distance apart within the field of view, as per

Table 2 Specimen details and testing parameters

\begin{tabular}{llllll}
\hline $\begin{array}{l}\text { Specimen } \\
\text { number }\end{array}$ & $\begin{array}{l}\text { Silicone } \\
\text { batch }\end{array}$ & $\begin{array}{l}\text { Testing } \\
\text { session }\end{array}$ & $\begin{array}{l}\text { Testing } \\
\text { speed }(\mathrm{mm} / \mathrm{min})\end{array}$ & $\begin{array}{l}\text { Grip } \\
\text { length }(\mathrm{mm})\end{array}$ & $\begin{array}{l}\text { Number } \\
\text { of tests }\end{array}$ \\
\hline $\mathbf{1}$ & 1 & 1 & 30 & 30 & 3 \\
$\mathbf{2}$ & 1 & 1 & 30 & 30 & 3 \\
$\mathbf{3}$ & 1 & 1 & 30 & 30 & 3 \\
$\mathbf{4}$ & 1 & 1 & 30 & 20 & 2 \\
$\mathbf{5}$ & 1 & 2 & 30 & 30 & 3 \\
$\mathbf{6}$ & 1 & 2 & 30 & 20 & 3 \\
$\mathbf{7}$ & 2 & 2 & 30 & 30 & 3 \\
$\mathbf{8}$ & 2 & 2 & 30 & 20 & 3 \\
\hline
\end{tabular}




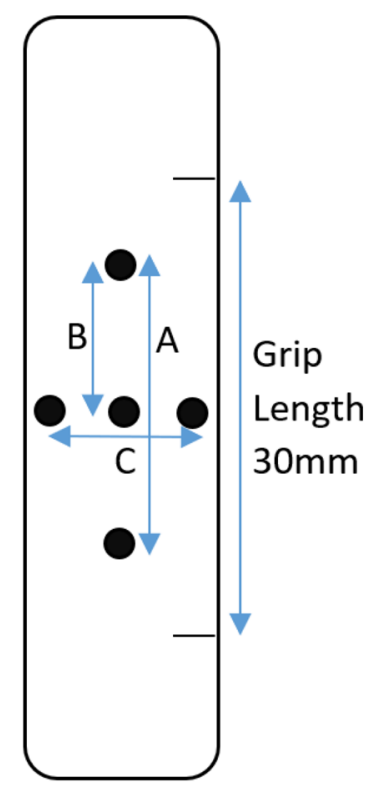

(a)

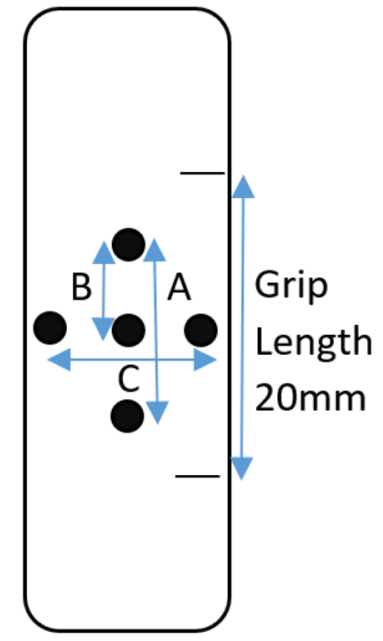

(b)

Fig. 3 Schematic of silicone specimens with marker positions for a $30 \mathrm{~mm}$ of grip length, where A is $20 \mathrm{~mm}, \mathrm{~B}$ is $10 \mathrm{~mm}$ and C is $7 \mathrm{~mm}$, and $\mathbf{b}$ a 20 -mm grip length, where A is $10 \mathrm{~mm}, \mathrm{~B}$ is $5 \mathrm{~mm}$, and $\mathrm{C}$ is $7 \mathrm{~mm}$

the manufacturer's instructions. When processing the captured video, a digital strain gauge was placed between two marker points. Specimens were marked using a permanent marker in the positions indicated in Fig. 3, so the digital gauge length could vary. An example of a marked silicone specimen is shown in Fig. 1.

When processing the data, the target size for each marker was varied. The target area defines the area in which the software locates the surface marker. Additionally, within the software, the user has the option to control certain elements of the tracking algorithm, for example, in order to account for the stretch and rotation in specimens subjected to large deformations. The post-processing variations are outlined in Table 3, and were allowed for the purpose of accounting for varying user preferences.

The data values measured and recorded during our experimental tests capture the inherent variation between the constitutive behaviour of the different specimens listed in Table 2. The values for the applied force versus maximum vertical displacement in tensile loading for the individual specimens are shown in Fig. 4. Note that, for the rubber-like material, the force needed to be increased to a critical level before non-zero macroscopic deformations could be observed. For the specimens in each batch, the data are represented together in Fig. 5 where the average values, which are typically used for the calibration of deterministic models, are marked by red lines. In this figure, different quantities of interest are also illustrated. Specifically, in addition to the applied force versus maximum vertical displacement observed experimentally, we have calculated the following nonlinear quantities:

- The first Piola-Kirchhoff (PK) tensile stress, representing the force per unit area in the reference configuration,

$$
P=\frac{F}{A}
$$

where $F$ is the applied tensile force and $A=40 \mathrm{~mm}^{2}$ is the cross-sectional area.

Table 3 Post-processing parameters for the optical strain measurement system, with the gauge lengths as indicated in Fig. 3

\begin{tabular}{lllll}
\hline & Settings 1 & Settings 2 & Settings 3 & Settings 4 \\
\hline Tracking algorithm & Deform only & Stretch, rotate and deform & Deform only & Deform only \\
Target 1 size & Random (based on user) & Random (based on user) & $45 \times 45$ pixels & $45 \times 45$ pixels \\
Target 2 size & Random (based on user) & Random (based on user) & $45 \times 45$ pixels & $45 \times 45$ pixels \\
Gauge length & A & A & A & B \\
\hline
\end{tabular}


Fig. 4 Experimental data for applied force versus maximum vertical displacement in tensile loading of individual silicone specimens listed in Table 2
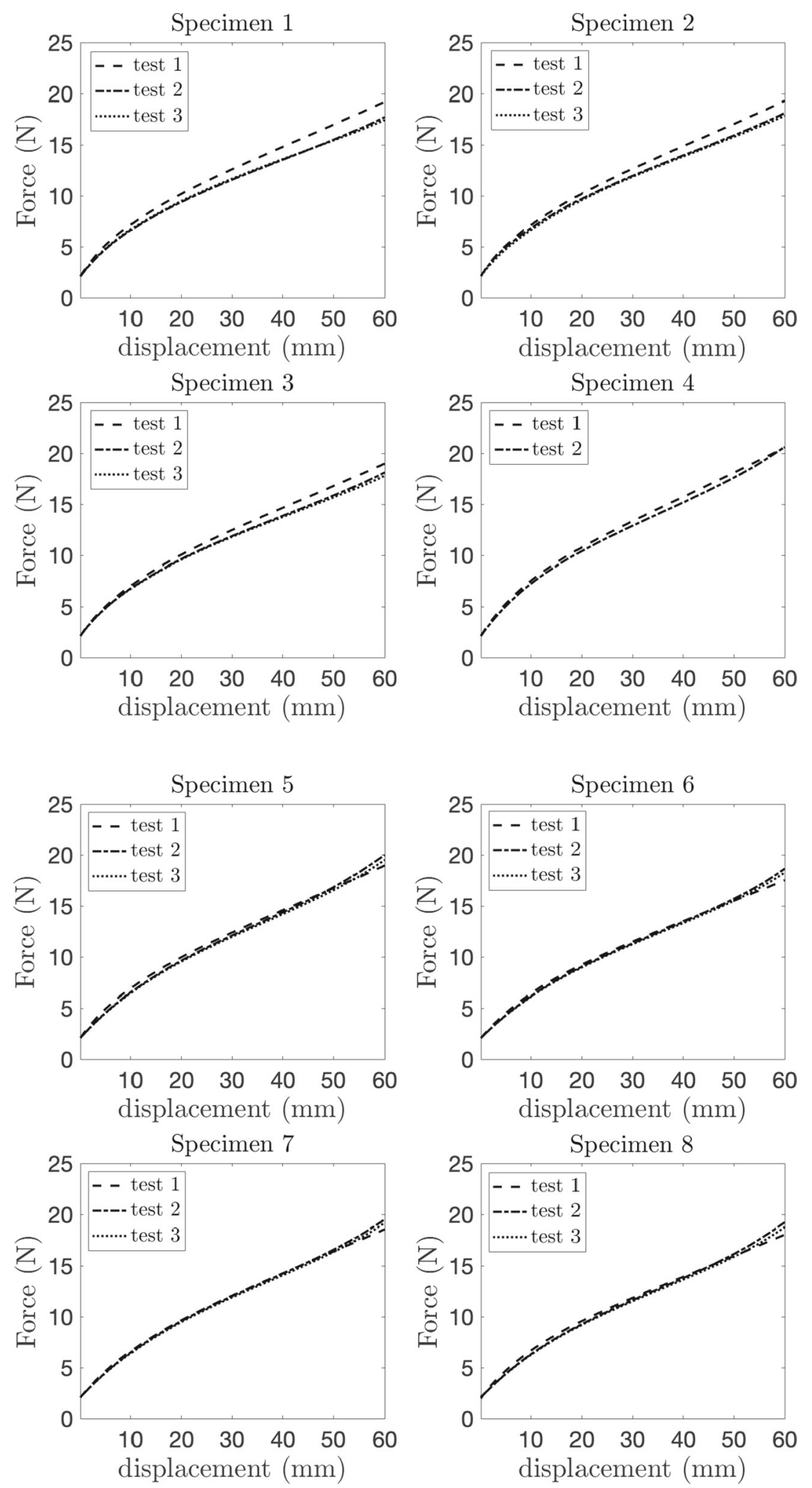

- The nonlinear stretch modulus [36]

$$
E=\frac{T}{\ln a}=\frac{a P}{\ln a},
$$


Fig. 5 Experimental data for applied force versus maximum vertical displacement in tensile loading of the two batches of silicone specimens listed in Table 2, together with the first Piola-Kirchhoff (PK) tensile stress given by Eq. 1, and the nonlinear stretch and shear moduli defined by Eqs. 2 and 3, respectively. The red lines indicate the arithmetic mean data values
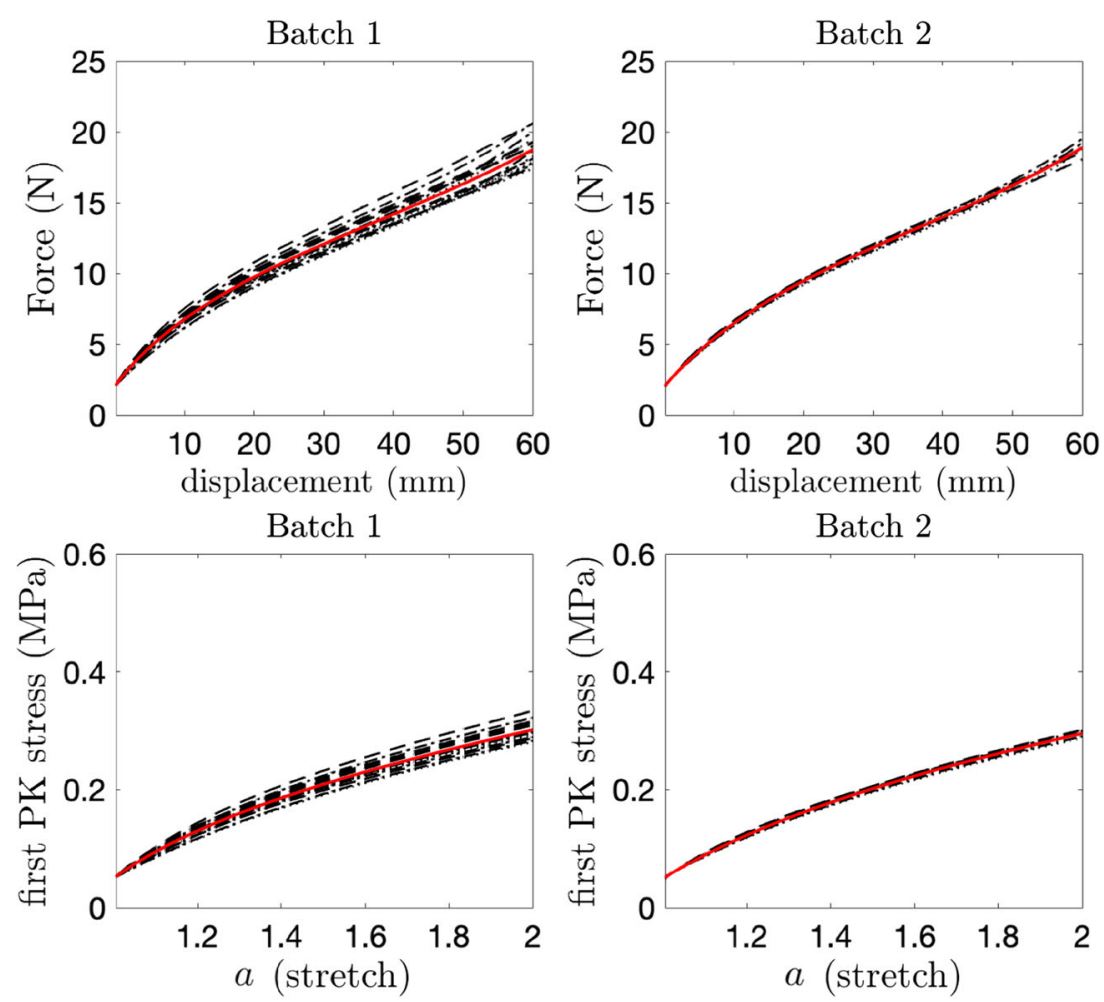

Batch 1
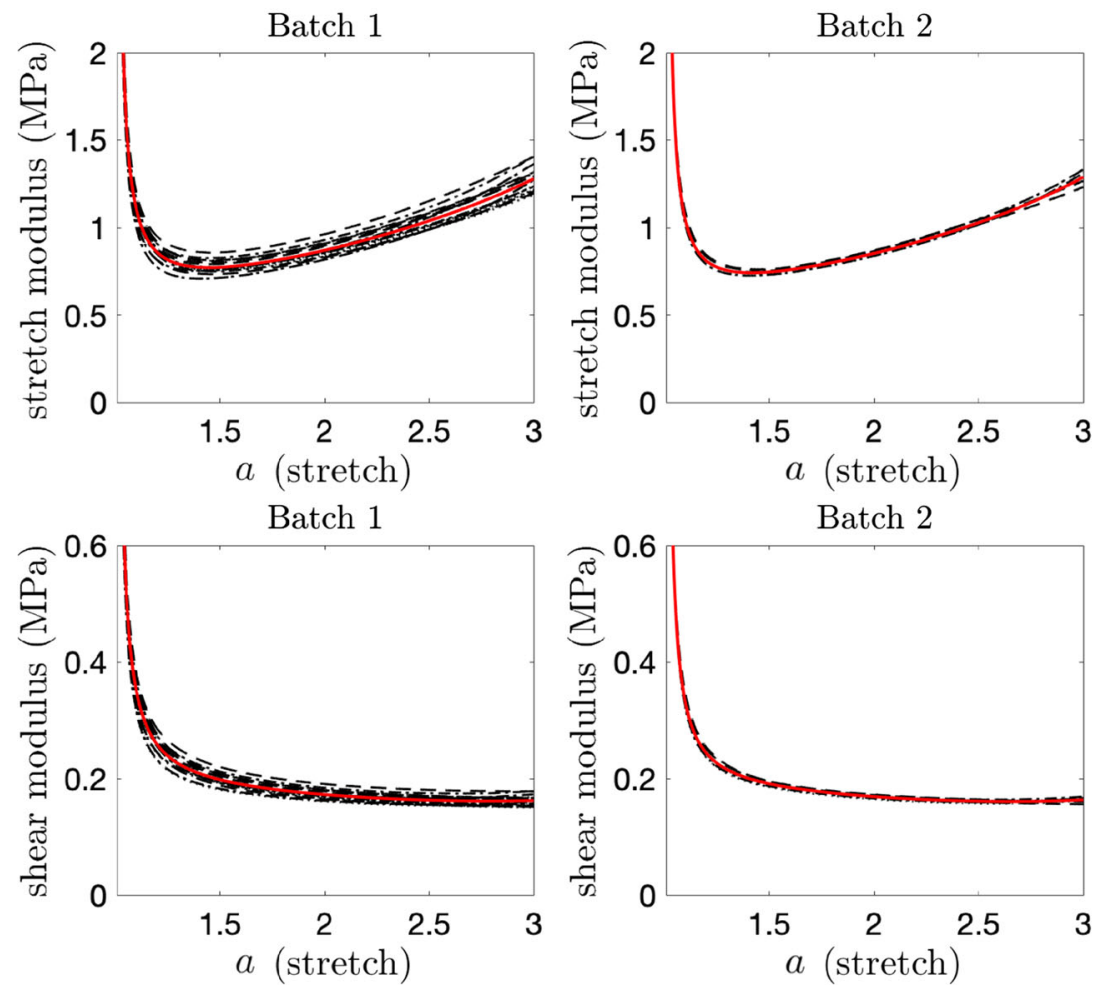

where $a$ is the stretch ratio and $T=a P$ is the Cauchy stress (representing the force per unit area in the current configuration), with $P$ the first Piola-Kirchhoff stress given by Eq. 1.

- The nonlinear shear modulus, given by the universal formula [36]

$\mu=\frac{E \ln a}{a^{2}-a^{-1}}=\frac{a P}{a^{2}-a^{-1}}$, 
where $E$ is the stretch modulus defined by Eq. 2 .

For formal definitions and explicit derivations of key nonlinear elastic moduli in homogeneous isotropic finite elasticity, and their universal relations under large strains, we refer to [36].

\section{Stochastic modelling}

Next, we construct specific stochastic homogeneous hyperelastic models where the parameters are characterised by spatially independent probability distributions and optimised to the collected data.

\subsection{Stochastic isotropic incompressible hyperelastic models}

We recall that a homogeneous hyperelastic material is defined by a strain-energy function $W(\mathbf{F})$, depending on the deformation gradient, $\mathbf{F}$, with respect to a reference configuration $[15,48,76]$. Traditionally, this is characterised by a set of deterministic model parameters, which contribute to the constant elastic moduli under small strains, or to the nonlinear elastic moduli, which are functions of the deformation under large strains [36]. In contrast, a stochastic hyperelastic model has parameters which are defined by probability density functions [37, 65-67]. Typically, each model parameter is described in terms of its mean value and its variance, which hold information regarding the range of values about the mean value [5, $6,18,29,44]$. Here, we construct stochastic homogeneous isotropic incompressible hyperelastic models that rely on the following assumptions [33-35, 37, 38, 40]:

(A1) Material objectivity, stating that constitutive equations must be invariant under changes of frame of reference. This requires that the scalar strain-energy function, $W=W(\mathbf{F})$, depending only on the deformation gradient $\mathbf{F}$, with respect to the reference configuration, is unaffected by a superimposed rigid-body transformation (which involves a change of position) after deformation, i.e., $W\left(\mathbf{R}^{T} \mathbf{F}\right)=W(\mathbf{F})$, where $\mathbf{R} \in S O(3)$ is a proper orthogonal tensor (rotation). Material objectivity is guaranteed by defining strain-energy functions in terms of the scalar invariants.

(A2) Material isotropy, requiring that the strain-energy function is unaffected by a superimposed rigid-body transformation prior to deformation, i.e., $W(\mathbf{F Q})=W(\mathbf{F})$, where $\mathbf{Q} \in S O(3)$. For isotropic materials, the strain-energy function is a symmetric function of the principal stretch ratios $\left\{\lambda_{i}\right\}_{i=1,2,3}$ of $\mathbf{F}$, i.e., $W(\mathbf{F})=$ $\mathcal{W}\left(\lambda_{1}, \lambda_{2}, \lambda_{3}\right)$.

(A3) Baker-Ericksen (BE) inequalities, which state that the greater principal (Cauchy) stress occurs in the direction of the greater principal stretch [2, 28],

$\left(T_{i}-T_{j}\right)\left(\lambda_{i}-\lambda_{j}\right)>0 \quad$ if $\quad \lambda_{i} \neq \lambda_{j}, \quad i, j=1,2,3$,

where $\left\{\lambda_{i}\right\}_{i=1,2,3}$ and $\left\{T_{i}\right\}_{i=1,2,3}$ denote the principal stretches and principal Cauchy stresses, respectively, such that

$T_{i}=\lambda_{i} \frac{\partial \mathcal{W}}{\partial \lambda_{i}}, \quad i=1,2,3$.

In Eq. 4, the strict inequality ' $>$ ' is replaced by " $\geq$ " if any two principal stretches are equal.

(A4) For any given finite deformation, at any point in the material, the shear modulus, $\mu$, and its inverse, $1 / \mu$, are second-order random variables, i.e., they have finite mean value and finite variance [65-69].

Assumptions (A1)-(A3) are general principles in isotropic finite elasticity [15, 36, 48, 76]. In particular, for a homogeneous hyperelastic body under uniaxial tension, the deformation is a simple extension in the direction of tensile force if and only if the BE inequalities Eq. 4 hold [28]. Assumption (A3) implies that the shear modulus is always positive, i.e., $\mu>0$ [36], while (A4) places random variables at the foundation of hyperelastic models [12, 21, 37, 43, 62].

Assumption (A4) is guaranteed by setting the following mathematical expectations:

$\left\{\begin{array}{l}E[\mu]=\mu>0, \\ E[\log \mu]=\nu, \text { such that }|\nu|<+\infty\end{array}\right.$

By the maximum entropy principle $[19,20]$, under the constraints (6), the random shear modulus, $\mu$, with mean value $\mu$ and standard deviation $\|\mu\|=\sqrt{\operatorname{Var}[\mu]}$ (defined as the square root of the variance, $\operatorname{Var}[\mu]$ ), follows a gamma distribution 
(the maximum entropy distribution) [58-60,62], with shape and scale parameters $\rho_{1}>0$ and $\rho_{2}>0$ respectively, satisfying

$\underline{\mu}=\rho_{1} \rho_{2}, \quad\|\mu\|=\sqrt{\rho_{1}} \rho_{2}$.

The probability density function then takes the form $[1,16,25]$

$g\left(\mu ; \rho_{1}, \rho_{2}\right)=\frac{\mu^{\rho_{1}-1} e^{-\mu / \rho_{2}}}{\rho_{2}^{\rho_{1}} \Gamma\left(\rho_{1}\right)}, \quad$ for $\mu>0$ and $\rho_{1}, \rho_{2}>0$,

where $\Gamma: \mathbb{R}_{+}^{*} \rightarrow \mathbb{R}$ is the complete gamma function

$\Gamma(z)=\int_{0}^{+\infty} t^{z-1} e^{-t} \mathrm{~d} t$.

The word 'hyperparameters' is also used for $\rho_{1}$ and $\rho_{2}$ to distinguish them from $\mu$ and other material constants [62, p. 8].

In practice, elastic moduli can take on different values, corresponding to possible outcomes of experimental tests. The maximum entropy principle allows us to explicitly construct the probability laws of the model parameters, given the available information. Approaches for the explicit derivation of probability distributions for the elastic parameters of stochastic homogeneous isotropic hyperelastic models calibrated to experimental data for rubber-like material and soft tissues were proposed in [37, 67].

\subsection{Hypothesis testing}

Before, we attempted to construct models based on the collected data, we applied standard statistical tests [11] to verify whether we can treat the entire dataset as one, or we must model the dataset of each batch separately. First, we used an unpaired $t$ test [52] to compare the shear modulus data at small strain for the two batches. This test provided a $p$ value of approximately $10^{-12}$, which was less than the 'standard' 0.05 significance level, and therefore allowed us to reject the null hypothesis that the moduli of the two batches come from the same distribution. The significant differences between the data corresponding to the two batches are illustrated in Fig. 6 . Further, we used a $\chi^{2}$ (chi-square) goodness-of-fit test [51] to check that we cannot reject the null hypothesis that the shear modulus data of each batch come from a gamma distribution. The $p$ values for the batch 1 and 2 fits were 0.1 and 0.07 , respectively. Thus, we could not reject the hypothesis that the shear moduli are gamma-distributed.

Figure 7 illustrates the gamma and normal (Gaussian) probability distributions fitted to the shear modulus data at small strain. We note the similarity between the represented gamma and normal distributions. This is not accidental because, when $\rho_{1} \rightarrow \infty$, the gamma probability distribution Eq. 8 is approximated by a normal distribution (see Appendix A for a detailed proof). Table 4 provides the fitted variables for both distributions. As we see, $\rho_{1}$ is very large compared to $\rho_{2}$, justifying the

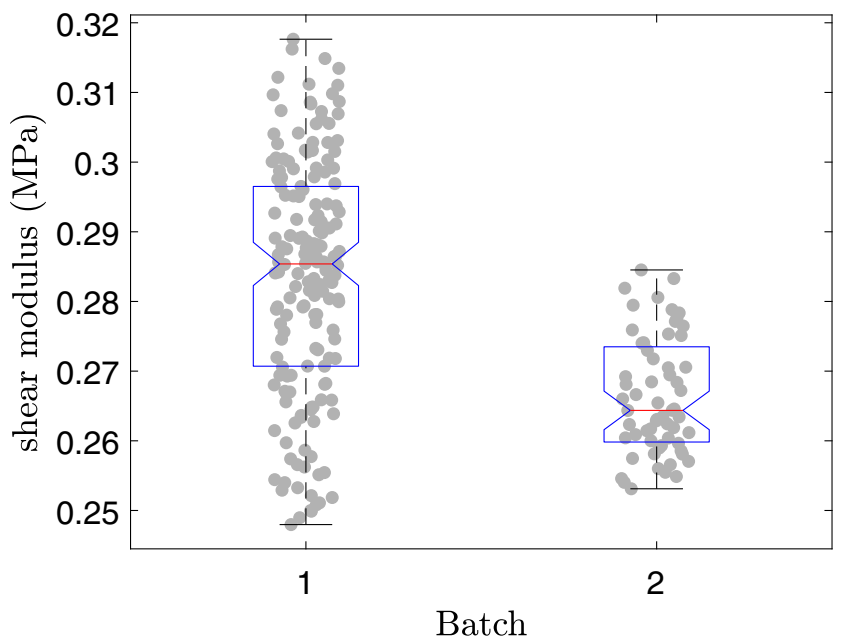

Fig. 6 Box plots of the shear modulus at small strain data of the two batches. On each box, the grey dots represent the data, the central mark indicates the median, and the bottom and top edges of the box indicate the 25 th and 75 th percentiles, respectively. The whiskers extend to the most extreme data points. Critically, these distributions are significantly different 
Fig. 7 Probability distributions derived from the data values for the random shear modulus at small strain. The parameters for these distributions are recorded in Table 4
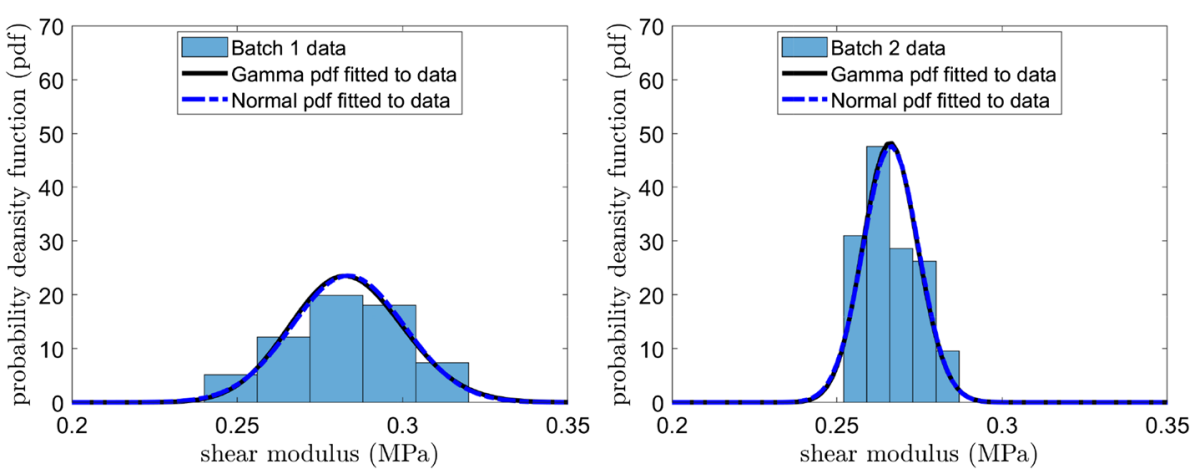

similarity between the two distributions. However, elastic moduli cannot be characterised by the normal distribution since this distribution is defined on the entire real line whereas elastic moduli are typically positive. Thus, the gamma distribution is chosen for its consistency with the mechanical properties of elastic materials.

\subsection{Stochastic calibration}

Applying the stochastic method developed in [37], we specialise to three different stochastic homogeneous isotropic strainenergy functions which we calibrate to the collected data. These strain-energy functions are presented in Table 5, while their constitutive parameters fitted to the experimental measurements for batch 1 and batch 2 are recorded in Table 6. Note that the calculated shear moduli are comparable to those recorded in Table 4. The load-deformation results are plotted in Figs. 8 and 9, demonstrating that all three models perform well, but exhibit different levels of accuracy when compared to the actual data.

\subsection{Bayesian model selection}

In this section, we employ Bayes' theorem $[3,30]$ to select the best perfoming model. We denote by $P(M)$ the prior probability of a model $M$ before the data values $D$ are taken into account, and by $P(D \mid M)$ the likelihood, or the probability of obtaining the data values $D$ from the model $M$. Bayes' theorem states that

$P(M \mid D)=\frac{P(M) P(D \mid M)}{P(D)}$,

where $P(M \mid D)$ is the posterior probability of the model $M$, and $P(D)$ is the normalisation value, known as the marginal likelihood. This theorem provides also a methodology for estimating the odds for a model $M^{(i)}$ to another model $M^{(j)}$ in light of the data $D$, i.e.,

$O_{i j}=\frac{P\left(M^{(i)} \mid D\right)}{P\left(M^{(j)} \mid D\right)}=\frac{P\left(M^{(i)}\right) P\left(D \mid M^{(i)}\right)}{P\left(M^{(j)}\right) P\left(D \mid M^{(j)}\right)}=\frac{P\left(M^{(i)}\right)}{P\left(M^{(j)}\right)} B_{\mathrm{ij}}$,

where

$B_{i j}=\frac{P\left(D \mid M^{(i)}\right)}{P\left(D \mid M^{(j)}\right)}$

is known as the Bayes factor. Formula (11) states that the posterior odds $O_{\mathrm{ij}}$ for the model $M^{(i)}$ against the model $M^{(j)}$, given the data $D$, are equal to the prior odds multiplied by the Bayes factor. In particular, if the models have equal prior

Table 4 Parameters of the probability distributions derived from the data values for the random shear modulus at small strain

\begin{tabular}{lllll}
\hline Probability density function (pdf) & $\underline{\mu}$ & $\|\mu\|$ & $\rho_{1}$ & $\rho_{2}$ \\
\hline Gamma pdf fitted to batch 1 data & 0.2837 & 0.0171 & 275.4403 & 0.0010 \\
Normal pdf fitted to batch 1 data & 0.2837 & 0.0170 & - & - \\
Gamma pdf fitted to batch 2 data & 0.2663 & 0.0083 & 1029.4047 & 0.0003 \\
Normal pdf fitted to batch 2 data & 0.2663 & 0.0084 & - & - \\
\hline
\end{tabular}


Table 5 Incompressible isotropic hyperelastic models, $\mathcal{W}\left(\lambda_{1}, \lambda_{2}, \lambda_{3}\right)$, their nonlinear shear modulus $\mu(a)$ at a given stretch $a$, and the shear modulus at infinitesimal deformation, $\lim _{a \rightarrow 1} \mu(a)=\bar{\mu}$

\begin{tabular}{lll}
\hline Material model & $\begin{array}{l}\text { Strain-energy density } \\
\mathcal{W}\left(\lambda_{1}, \lambda_{2}, \lambda_{3}\right)\end{array}$ & Shear moduli \\
\hline Mooney-Rivlin & $\frac{C_{1}}{2}\left(\lambda_{1}^{2}+\lambda_{2}^{2}+\lambda_{3}^{2}-3\right)+\frac{C_{2}}{2}\left(\lambda_{1}^{-2}+\lambda_{2}^{-2}+\lambda_{3}^{-2}-3\right)$ & $\mu(a)=C_{1}+\frac{C_{2}}{a}$ \\
{$[42,54]$} & $C_{1}, C_{2}$ independent of deformation & $\bar{\mu}=C_{1}+C_{2}$ \\
Gent-Gent & $-\frac{C_{1}}{2 \beta} \ln \left[1-\beta\left(\lambda_{1}^{2}+\lambda_{2}^{2}+\lambda_{3}^{2}-3\right)\right]+\frac{3 C_{2}}{2} \ln \frac{\lambda_{1}^{-2}+\lambda_{2}^{-2}+\lambda_{3}^{-2}}{3}$ & $\mu(a)=\frac{C_{1}}{1-\beta\left(a^{2}+2 / a-3\right)}+\frac{3 C_{2}}{2 a^{2}+1 / a}$ \\
{$[49,53]$} & $C_{1}, C_{2}, \beta$ independent of deformation & $\bar{\mu}=C_{1}+C_{2}$ \\
Ogden & $\sum_{p=1}^{3} \frac{C_{p}}{2 \alpha_{p}^{2}}\left(\lambda_{1}^{2 \alpha_{p}}+\lambda_{2}^{2 \alpha_{p}}+\lambda_{3}^{2 \alpha_{p}}-3\right)$ & $\mu(a)=\sum_{p=1}^{3} \frac{C_{p}}{\alpha_{p}} \frac{a^{1-\alpha_{p}}\left(1-a^{3 \alpha_{p}}\right)}{1-a^{3}}$ \\
{$[47]$} & $C_{p}$ independent of deformation; $\alpha_{1}=1, \alpha_{2}=-1, \alpha_{3}=-2$, & $\bar{\mu}=\sum_{p=1}^{3} C_{p}$ \\
\hline
\end{tabular}

probabilities, $P\left(M^{(i)}\right)=P\left(M^{(j)}\right)$, i.e., there is no prior favourite, then, by Eq. 11, the posterior odds are equal to the Bayes factor, i.e., $O_{\mathrm{ij}}=B_{\mathrm{ij}}$.

However, if the Bayes factor is equal to 1, then Occam's razor [22-24, 73] would imply that a larger prior probability should be assigned to the simpler model than to the more complex one for reasons of parsimony.

Maintaining a general framework, we assume $P(D \mid M)$ to be an arbitrary probability that is symmetric about the mean value $D=0$ and decreasing in the absolute value of $D$ (see also the imaginative cartoon by D. M. Titterington (1982) [74]). In this case, the Bayes factor $B_{\mathrm{ij}}$ satisfies the inequality as follows [4]:

$B_{\mathrm{ij}} \geq \frac{\left\|D^{(j)}\right\|+\sqrt{2 \ln \left(\left\|D^{(j)}\right\|+1.2\right)}}{e^{\left\|D^{(i)}\right\|^{2} / 2}} \sqrt{\frac{2}{\pi}}$,

where $\left\|D^{(i)}\right\|$ and $\left\|D^{(j)}\right\|$ designate the standard deviation that the predicted quantity of interest computed with the model $M^{(i)}$ and $M^{(j)}$, respectively, deviates from the observed data value $D$. The formula for calculating $\left\|D^{(i)}\right\|$ is as follows,

$\left\|D^{(i)}\right\|=\frac{\left|\underline{Q}^{(i)}-\underline{D}\right|}{\|D\|}$,

where, for the quantity of interest, $\underline{Q}^{(i)}$ is the expected value computed with the model $M^{(i)}$, and $\underline{D}$ and $\|D\|$ represent the experimentally observed mean value and standard deviation, respectively. The expression on the right-hand side of Eq. 13 provides an explicit lower bound on the Bayes factor $B_{\mathrm{ij}}$. Thus, taking equal prior probabilities, the lower bound on the Bayes factor given by Eq. 13 represents a lower bound on the posterior odds. This lower bound is an estimate of the amount of evidence against the model $M^{(i)}$, i.e., the maximum support for the model $M^{(j)}$ provided by the data.

Table 6 Parameters of stochastic constitutive models given in Table 5 calibrated to the data, and the corresponding random nonlinear shear modulus $\mu=\mu(a)$ at $a=1.15$

\begin{tabular}{|c|c|c|c|c|}
\hline \multirow[t]{2}{*}{ Stochastic model } & \multicolumn{2}{|c|}{$\begin{array}{l}\text { Calibrated parameters } \\
\text { (mean value } \pm \text { standard deviation) }\end{array}$} & \multicolumn{2}{|c|}{$\begin{array}{l}\text { Shear modulus } \\
\text { (mean value } \pm \text { standard deviation) }\end{array}$} \\
\hline & Batch 1 & Batch 2 & Batch 1 & Batch 2 \\
\hline Mooney-Rivlin & $\begin{array}{l}C_{1}=0.0936 \pm 0.0030 \\
C_{2}=0.1696 \pm 0.0115\end{array}$ & $\begin{array}{l}C_{1}=0.1029 \pm 0.0001 \\
C_{2}=0.1696 \pm 0.0115\end{array}$ & $\mu=0.2411 \pm 0.0130$ & $\mu=0.2277 \pm 0.0056$ \\
\hline Gent-Gent & $\begin{array}{l}C_{1}=0.0971 \pm 0.0042 \\
C_{2}=0.1826 \pm 0.0110 \\
\beta=0.0434\end{array}$ & $\begin{array}{l}C_{1}=0.1007 \pm 0.0011 \\
C_{2}=0.1625 \pm 0.0053 \\
\beta=0.0421\end{array}$ & $\mu=0.2532 \pm 0.0136$ & $\mu=0.2397 \pm 0.0056$ \\
\hline $\begin{array}{l}\text { Ogden } \\
\alpha_{1}=1, \alpha_{2}=-1, \alpha_{3}=-2\end{array}$ & $\begin{array}{l}C_{1}=-0.0645 \pm 0.0143 \\
C_{2}=-0.0764 \pm 0.0155 \\
C_{3}=0.4861 \pm 0.0534\end{array}$ & $\begin{array}{l}C_{1}=-0.0437 \pm 0.0111 \\
C_{2}=-0.0844 \pm 0.0112 \\
C_{3}=0.4505 \pm 0.0345\end{array}$ & $\mu=0.2719 \pm 0.0720$ & $\mu=0.2563 \pm 0.0272$ \\
\hline
\end{tabular}




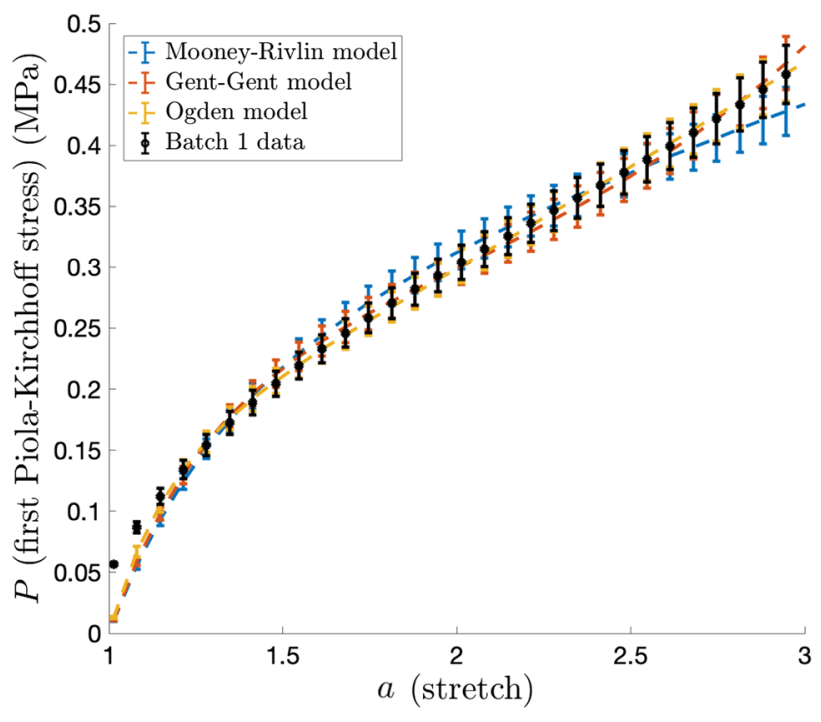

(a)

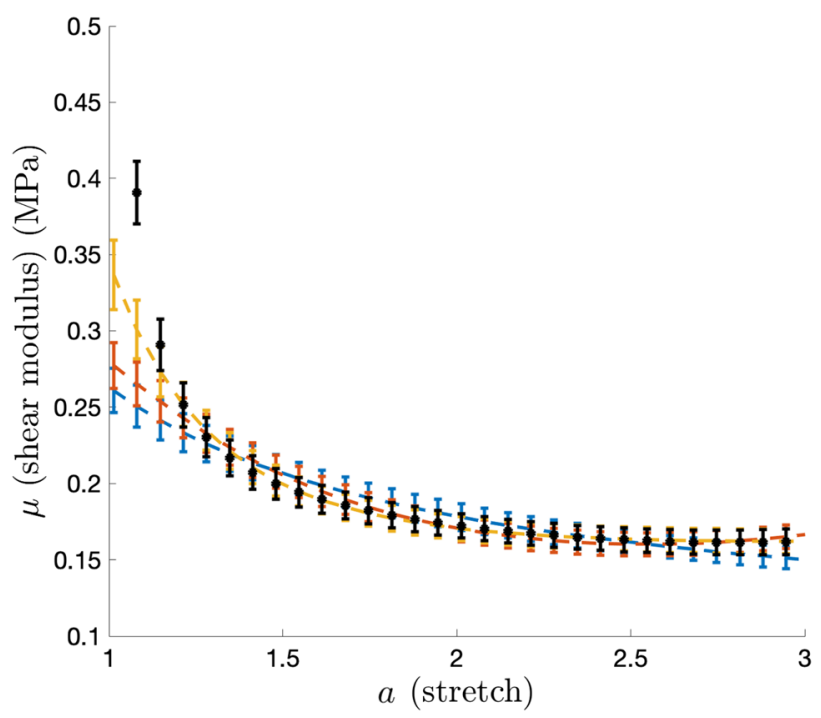

(c)

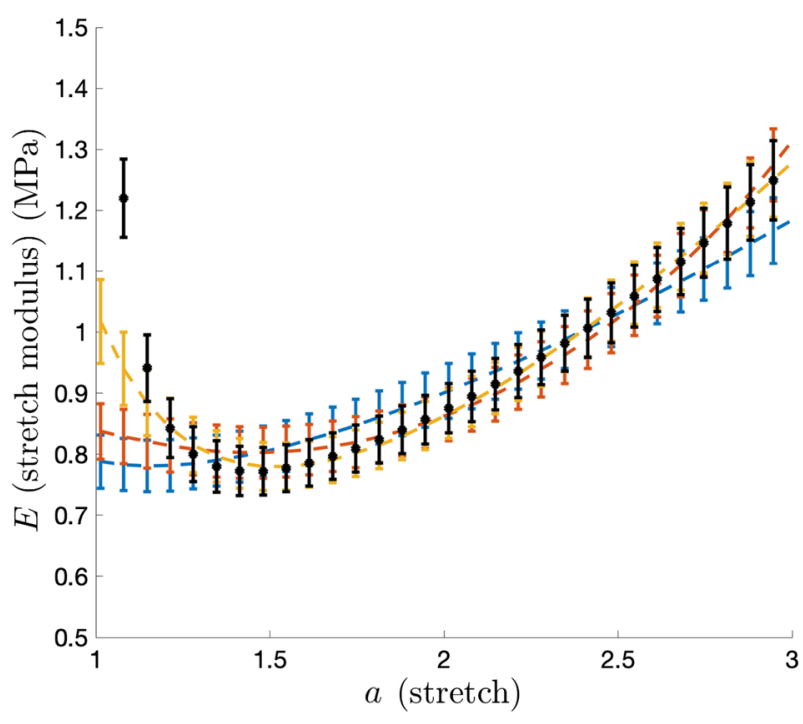

(b)

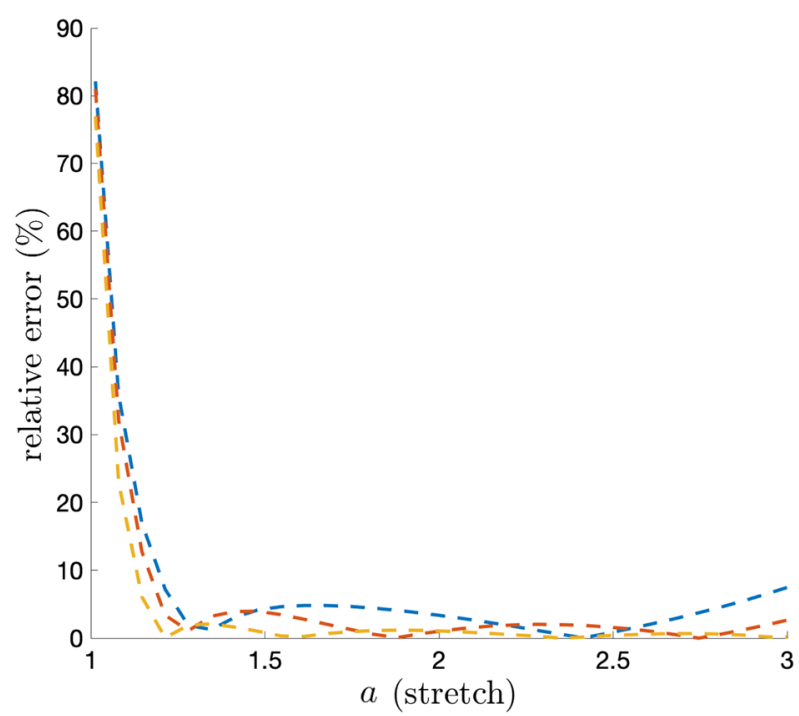

(d)

Fig. 8 Stochastic models calibrated to the batch 1 data, with the parameters recorded in Table 6, showing: a the first Piola-Kirchhoff tensile stress, $\mathbf{b}$ the nonlinear stretch modulus, $\mathbf{c}$ the nonlinear shear modulus, and $\mathbf{d}$ the relative error for the shear modulus mean values

We now apply these bounds to select the best performing model among the models calibrated to our experimental data. For example, at $a=1.15$, for each model recorded in Table 6 , we calculate the standard deviations that the mean shear modulus $\mu$ deviates from the experimental mean data value 0.2909 , given that the experimental standard deviation is 0.0170 . Applying formula (14), we obtain the following:

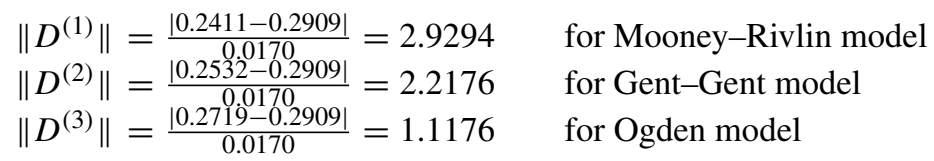

Assuming no prior favourite model (equal prior probabilities), by Eq. 13, it follows that the Bayes factors (or the odds) for the Ogden model against each of the other two models satisfy, respectively, $B_{31} \geq 1.9713$ and $B_{32} \geq 1.6174$. Next, 


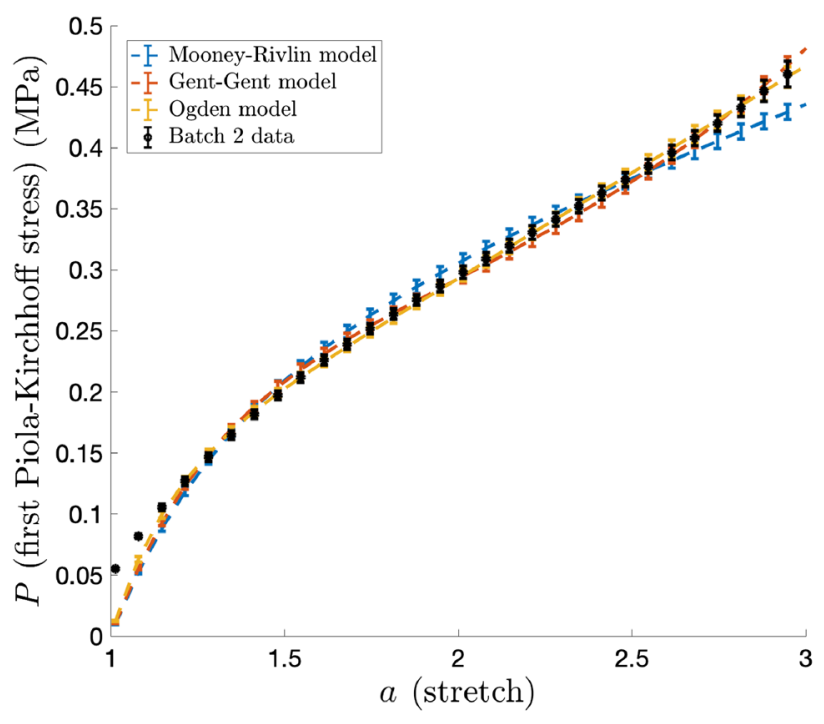

(a)

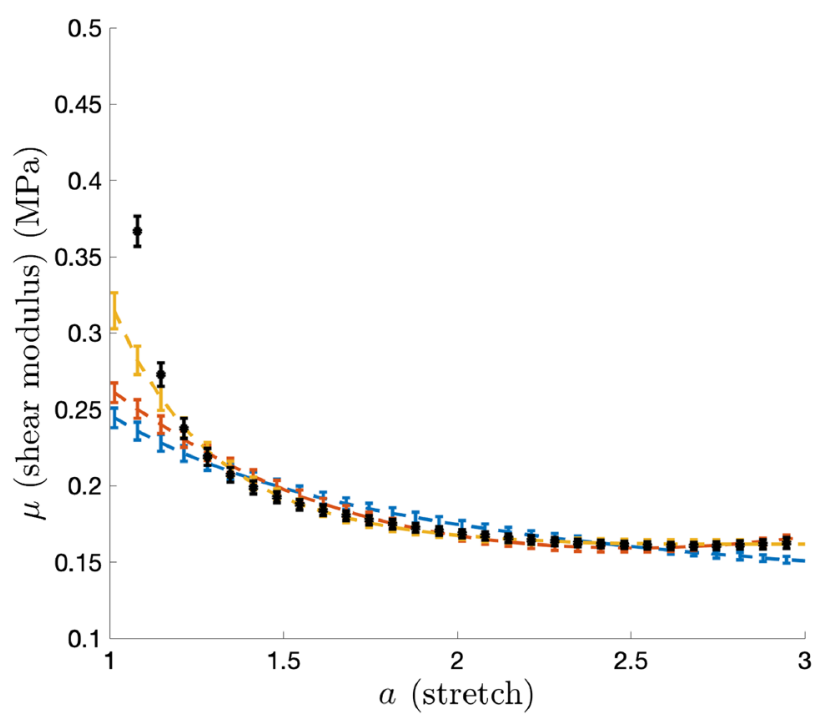

(c)

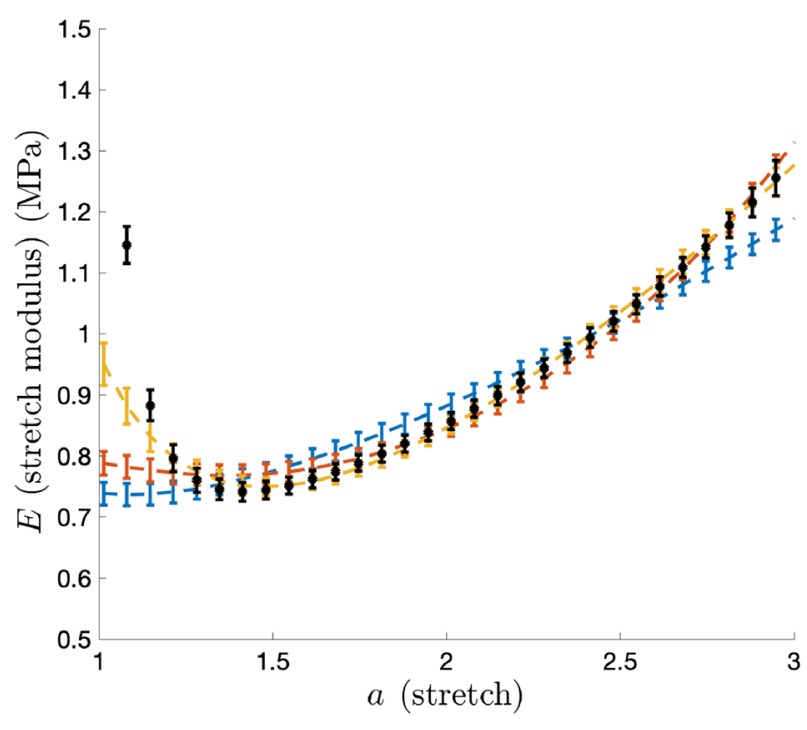

(b)

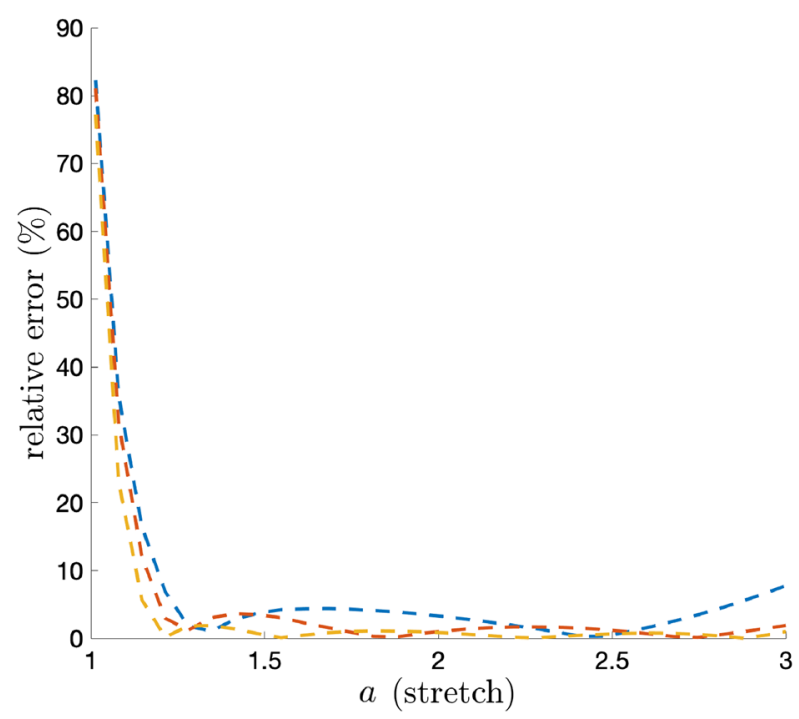

(d)

Fig. 9 Stochastic models calibrated to the batch 2 data, with the parameters recorded in Table 6, showing: a the first Piola-Kirchhoff tensile stress, (b) the nonlinear stretch modulus, $\mathbf{c}$ the nonlinear shear modulus, and $\mathbf{d}$ the relative error for the shear modulus mean values

taking $P\left(D \mid M^{(1)}\right)=1-P\left(D \mid M^{(3)}\right)$, by the lower bound on the Bayes factor $B_{31}$, the likelihood of obtaining the data with the Ogden model is $P\left(D \mid M^{(3)}\right) \geq 0.6634$. Similarly, assuming equal prior probabilities and taking $P\left(D \mid M^{(2)}\right)=$ $1-P\left(D \mid M^{(3)}\right)$, the lower bound on $B_{32}$ implies $P\left(D \mid M^{(3)}\right) \geq 0.6179$. Therefore, the data at $a=1.15$ are more likely to be reproduced by the Ogden model than by the other two models.

In Fig. 10, we illustrate the lower bounds on the Bayes factor $B_{\mathrm{ij}}$ for each model $M^{(i)}$ against another model $M^{(j)}$ at various stretch ratios. From these bounds, we infer that the Gent-Gent model is generally more likely to represent the data than the Mooney-Rivlin model, and the Ogden model is more likely than both the Mooney-Rivlin and the Gent-Gent models. These results are consistent with those in Figs. $8 \mathrm{~d}$ and $9 \mathrm{~d}$ where the relative errors for the mean values of the shear modulus are shown. The relative error was calculated in the usual way, i.e., $\left|\underline{\mu} \underline{m o d e l}-\underline{\mu}_{\text {data }}\right| / \underline{\mu}_{\text {data }}$, where $\underline{\mu}_{\text {model }}$ is the mean value of the shear modulus for the respective model and $\underline{\mu}_{\text {data }}$ is the mean value of the shear modulus calculated from the experimental data. 


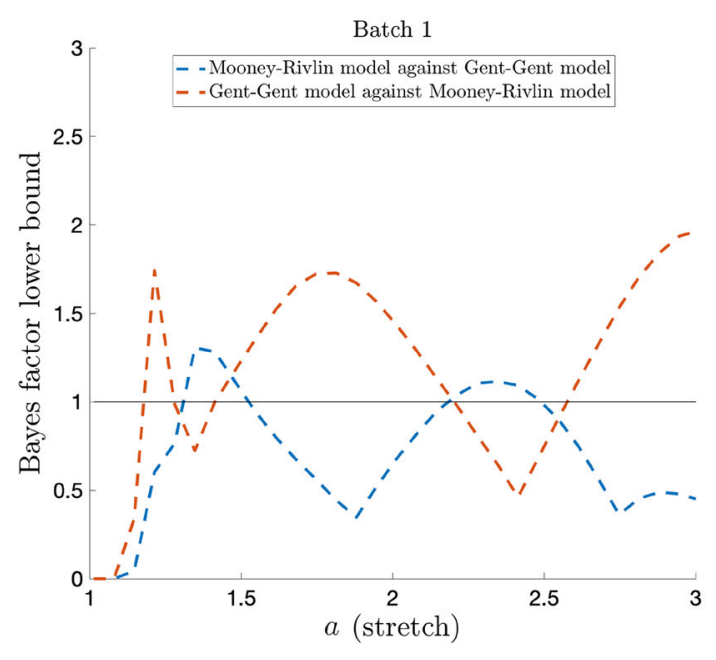

(a)

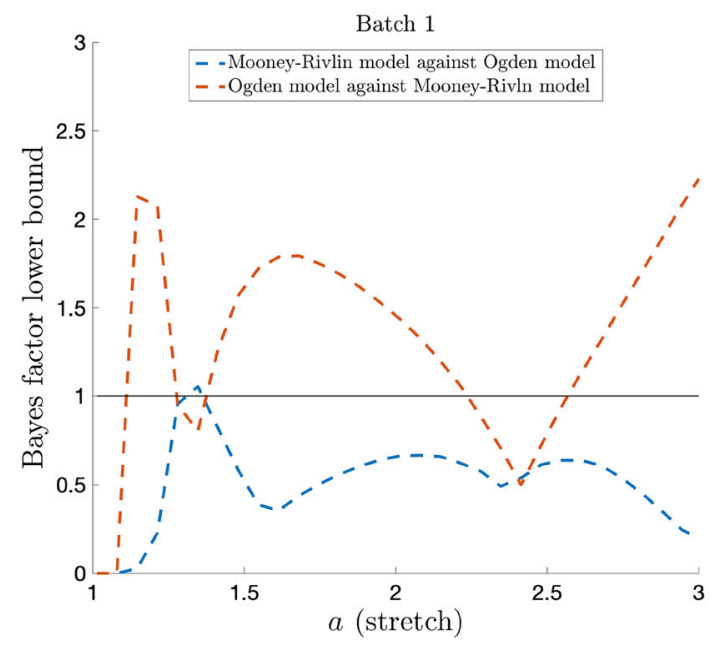

(c)

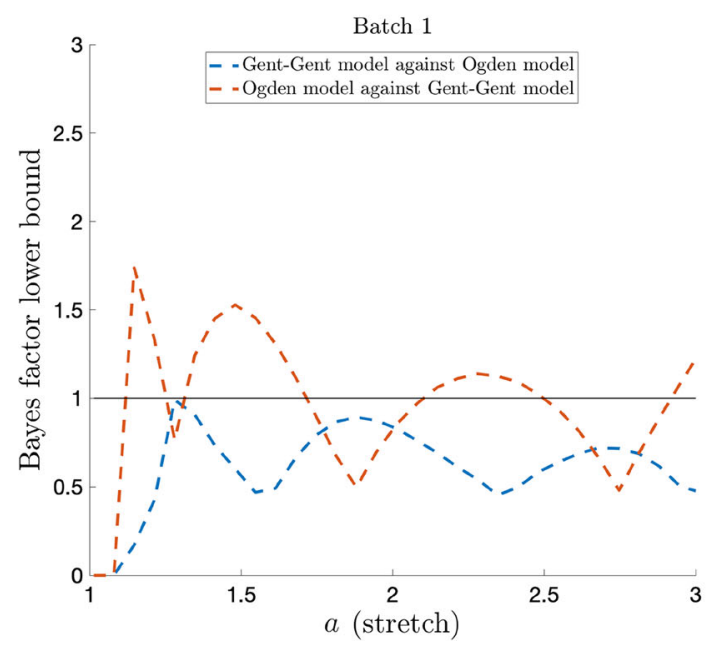

(e)

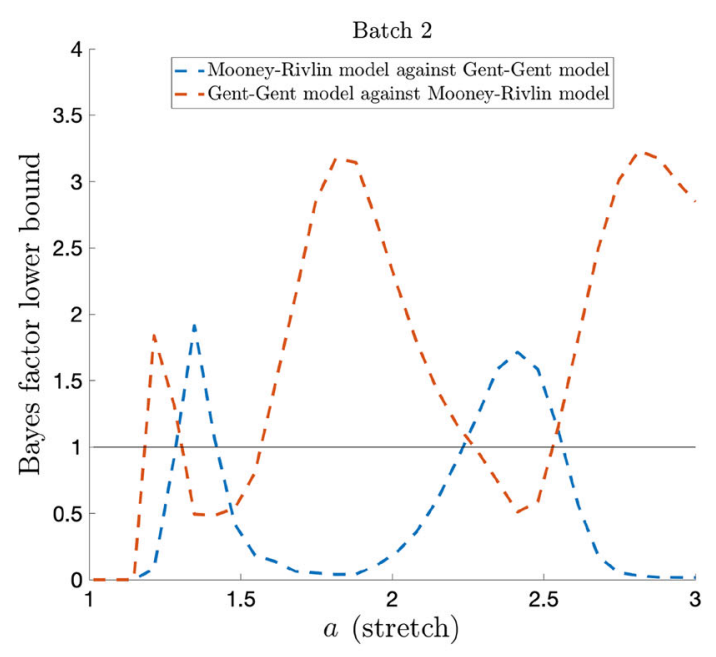

(b)

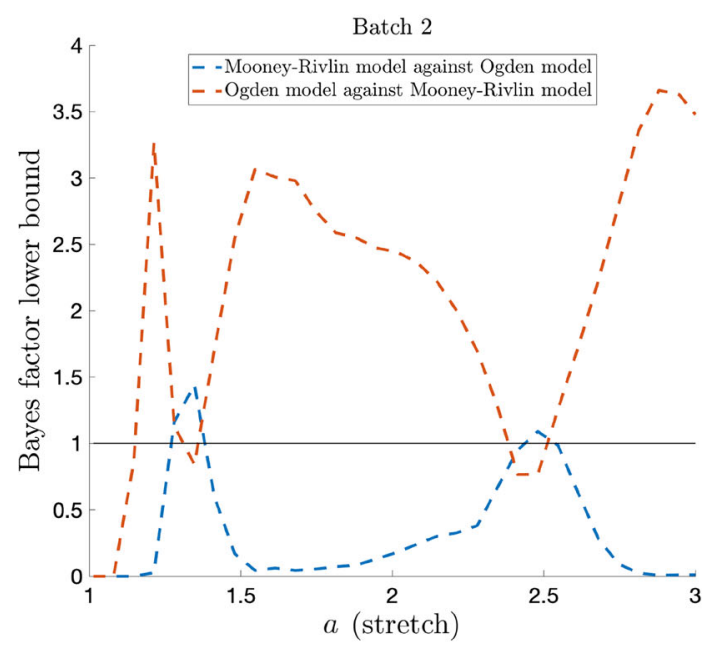

(d)

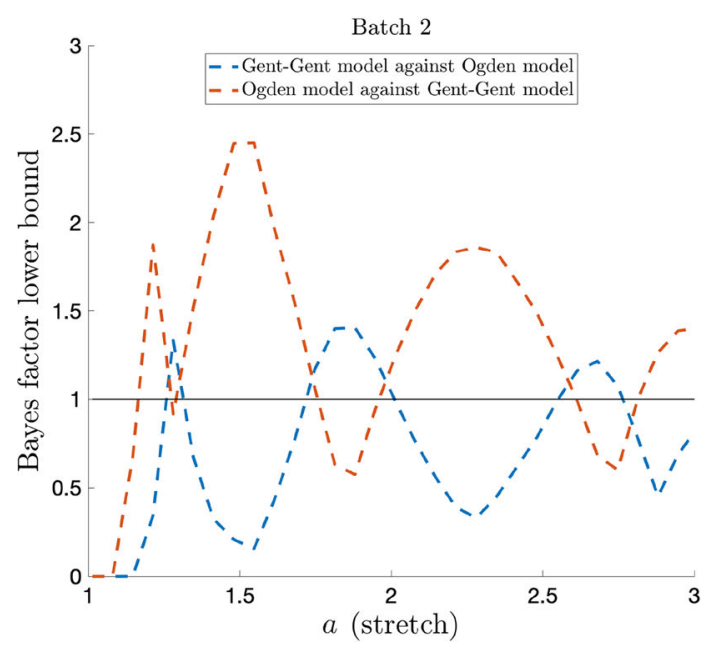

(f)

Fig. 10 Lower bounds on the Bayes factor for each model against the other at various stretch ratios 


\section{Conclusion}

We report on experimental tests on different samples of a manufactured rubber-like material under large tensile loading, and employ a stochastic strategy to derive constitutive models that take into account the variability in the collected data. Specifically, we construct isotropic incompressible hyperelastic models with model parameters defined as spatially independent random variables characterised by probability density functions at a continuum level. In addition, we provide a methodology where an explicit lower bound on the Bayes factor is used to compare different models, and then applied to select the model that is most likely to reproduce the data.

Ideally, these models are calibrated and validated on multiaxial test data [31, 32, 41, 63]. In this case also, our stochastic calibration and Bayesian model selection can be employed to obtain suitable models.

Our analysis is fully tractable mathematically and builds directly on knowledge from deterministic finite elasticity.

This study highlights the need for continuum models to consider the variability in the elastic behaviour of materials at large strains, and complements our previous theoretical investigations of how elastic solutions of fundamental problems in nonlinear elasticity can be extended to stochastic hyperelastic models [33-35, 38-40].

Acknowledgements The authors are grateful to Professor Nikolai Leonenko of Cardiff School of Mathematics for discussions on probability theory, and to Richard Thomas of Cardiff School of Engineering for his valuable support with the experimental testing and setup.

Funding information This study is supported by the Engineering and Physical Sciences Research Council of Great Britain under research grant EP/S028870/1 to L. Angela Mihai.

Data Accessability The datasets supporting this article have been uploaded as part of the Supplementary Material.

Open Access This article is distributed under the terms of the Creative Commons Attribution 4.0 International License (http://creativecommons. org/licenses/by/4.0/), which permits unrestricted use, distribution, and reproduction in any medium, provided you give appropriate credit to the original author(s) and the source, provide a link to the Creative Commons license, and indicate if changes were made.

\section{Appendix}

\section{A Normal distribution as limiting distribution of the gamma distribution}

Theorem A.1 The limiting distribution of the gamma distribution with shape and scale parameters $\rho_{1}$ and $\rho_{2}$, respectively, such that $\rho_{1} \rightarrow \infty$, is the Gaussian (normal) distribution with mean value $\rho_{1} \rho_{2}$ and standard deviation $\rho_{2} \sqrt{\rho_{1}}$.

Proof If $\mu$ is a random variable following a gamma probability distribution with shape parameter $\rho_{1}>0$ and scale parameter $\rho_{2}>0$, then,

$\underline{\mu}=\rho_{1} \rho_{2}, \quad\|\mu\|=\sqrt{\rho_{1}} \rho_{2}$,

where $\mu=E[\mu]$ is the mean value and $\|\mu\|=\sqrt{\operatorname{Var}[\mu]}$ is the standard deviation of $\mu$, with $\operatorname{Var}[\mu]$ denoting the variance of $\mu$. The moment generating function of $\mu$ is defined by the following:

$M_{\mu}(t)=E\left[e^{t \mu}\right]=\left(1-\rho_{2} t\right)^{-\rho_{1}}, \quad t<\frac{1}{\rho_{2}}$.

Subtracting the mean value $\underline{\mu}$ from $\mu$ and dividing the result by the standard deviation $\|\mu\|$ gives the following 1-1 transformation,

$Y=\frac{\mu-\underline{\mu}}{\|\mu\|}$,

or equivalently,

$Y=\frac{\mu}{\rho_{2} \sqrt{\rho_{1}}}-\sqrt{\rho_{1}}$.

The moment generating function of $Y$ is then

$M_{Y}(t)=E\left[e^{t Y}\right]=e^{-t \sqrt{\rho_{1}}} E\left[e^{t \frac{\mu}{\rho_{2} \sqrt{\rho_{1}}}}\right]=e^{-t \sqrt{\rho_{1}}}\left(1-\frac{t}{\sqrt{\rho_{1}}}\right)^{-\rho_{1}}, \quad t<\sqrt{\rho_{1}}$. 
Thus, the limiting moment generating function of $Y$ when $\rho_{1} \rightarrow \infty$ takes the form as follows:

$\lim _{\rho_{1} \rightarrow \infty} M_{Y}(t)=\lim _{\rho_{1} \rightarrow \infty} e^{-t \sqrt{\rho_{1}}}\left(1-\frac{t}{\sqrt{\rho_{1}}}\right)^{-\rho_{1}}, \quad-\infty<t<\infty$.

The above limit can be calculated as follows:

$\lim _{\rho_{1} \rightarrow \infty} e^{-t \sqrt{\rho_{1}}}\left(1-\frac{t}{\sqrt{\rho_{1}}}\right)^{-\rho_{1}}=\lim _{y=1 / \sqrt{\rho_{1}} \rightarrow 0_{+}} e^{-(t / y)}(1-t y)^{-1 / y^{2}}=e^{\lim _{y \rightarrow 0_{+}} \frac{-t y-\ln (1-t y)}{y^{2}}}$,

where, applying L'Hôspital's rule [27],

$\lim _{y \rightarrow 0_{+}} \frac{-t y-\ln (1-t y)}{y^{2}}=\lim _{y \rightarrow 0} \frac{-t+t /(1-t y)}{2 y}=\lim _{y \rightarrow 0_{+}} \frac{t^{2}}{2(1-t y)}=\frac{t^{2}}{2}$.

Therefore,

$\lim _{\rho_{1} \rightarrow \infty} M_{Y}(t)=e^{\frac{t^{2}}{2}}, \quad-\infty<t<\infty$,

which is the moment generating function of a normal-distributed random variable. Thus, the limiting distribution of a gamma distribution with $\rho_{1} \rightarrow \infty$ is the normal distribution. This completes the proof.

\section{References}

1. Abramowitz, M., Stegun, I.A.: Handbook of Mathematical Functions with Formulas, Graphs, and Mathematical Tables, National Bureau of Standards, Applied Mathematics Series, vol. 55, Washington (1964)

2. Baker, M., Ericksen, J.L.: Inequalities restricting the form of stress-deformation relations for isotropic elastic solids and Reiner-Rivlin fluids. J. Wash. Acad. Sci. 44, 24-27 (1954)

3. Bayes, T.: An essay toward solving a problem in the doctrine of chances. Philos. Trans. R. Soc. 53, 370-418 (1763)

4. Berger, J.O., Jefferys, W.H.: The application of robust Bayesian analysis to hypothesis testing and Occam's razor. J. Italian Stat. Soc. 1, 17-32 (1992)

5. Brewick, P.T., Teferra, K.: Uncertainty quantification for constitutive model calibration of brain tissue. J. Mech. Behav. Biomed. Mater. 85, 237-255 (2018)

6. Caylak, I., Penner, E., Dridger, A., Mahnken, R.: Stochastic hyperelastic modeling considering dependency of material parameters. Comput. Mech. 62, 1273-1285 (2018). https://doi.org/10.1007/s00466-018-1563-z

7. Criscione, J.C., Humphrey, J.D., Douglas, A.S., Hunter, W.C.: An invariant basis for natural strain which yields orthogonal stress response terms in isotropic hyperelasticity. J. Mech. Phys. Solids 48, 2445-2465 (2000)

8. Destrade, M., Saccomandi, G., Sgura, I.: Methodical fitting for mathematical models of rubber-like materials. Proc. R. Soc. A 473, 20160811 (2017)

9. Elishakoff, I., Soize C (eds.): Nondeterministic mechanics. Springer, New York (2012)

10. Evans, S.L., Holt, C.A.: Measuring the mechanical properties of human skin in vivo using digital image correlation and finite element modelling. J. Strain Anal. Eng. Des. 44, 337-345 (2009)

11. Freedman, D., Pusani, R., Perves, R. Statistics, 4th edn. W. W. Norton \& Company, New York (1991)

12. Freiling, C.: Axioms of symmetry: throwing darts at the real number line. J. Symbol. Log. 51(1), 190-200 (1986)

13. Geethama, V.G., Thomas, S.: Why does a rubber ball bounce? the molecular origins of rubber elasticity. Resonance 2(4), 48-54 (1997)

14. Ghanem, R., Higdon, D., Owhadi, H. (eds.): Handbook of Uncertainty Quantification. Springer, New-York (2017)

15. Goriely, A.: The mathematics and mechanics of biological growth. Springer, New York (2017)

16. Grimmett, G.R., Stirzaker, D.R. Probability and random processes, 3rd edn. Oxford University Press, Oxford (2001)

17. Hartmann, S.: Parameter estimation of hyperelasticity relations of generalized polynomial-type with constraint conditions. Int. J. Solids Struct. 38, 7999-8018 (2001)

18. Hughes, I., Hase, T.P.A.: Measurements and their uncertainties: a practical guide to modern error analysis. Oxford University Press, Oxford (2010)

19. Jaynes, E.T.: Information theory and statistical mechanics i. Phys. Rev. 108, 171-190 (1957)

20. Jaynes, E.T.: Information theory and statistical mechanics ii. Phys. Rev. 106, 620-630 (1957)

21. Jaynes, E.T.: Probability theory: the logic of science. Cambridge University Press, Cambridge (2003)

22. Jefferys, W.H., Berger, J.O.: Ockham's razor and Bayesian analysis. Am. Sci. 80, 64-72 (1992)

23. Jeffreys, H.: Some tests of significance, treated by the theory of probability. Math. Proc. Camb. Philos. Soc. 31, 203-222 (1935)

24. Jeffreys, H. Theory of probability, 3rd edn. Oxford University Press, Oxford, UK (1961)

25. Johnson, N.L., Kotz, S., Balakrishnan, N. Continuous univariate distributions, 2nd edn., vol. 1. Wiley, New York (1994)

26. Kaminski, M., Lauke, B.: Probabilistic and stochastic aspects of rubber hyperelasticity. Meccanica 53, 2363-2378 (2018)

27. L'Hôspital, G.F.A.: Analyse des infiniment petits, pour l'intelligence des lignes courbes. A Paris, de l'Imprimerie Royale, pp. 145-146 (1696) 
28. Marzano, M.: An interpretation of Baker-Ericksen inequalities in uniaxial deformation and stress. Meccanica 18, $233-235$ (1983)

29. McCoy, J.J.: A statistical theory for predicting response of materials that possess a disordered structure, Technical report ARPA 2181, AMCMS Code 5911.21.66022 Army Materials and Mechanics Research Center, Watertown, Massachusetts (1973)

30. McGrayne, S.B.: The Theory That Would Not Die: How Bayes' Rule Cracked the Enigma Code, Hunted Down Russian Submarines, an Emerged Triumphant from Two Centuries of Controversy Paperback ed. Yale University Press, New Haven (2012)

31. Mihai, L.A., Budday, S., Holzapfel, G.A., Kuhl, E., Goriely, A.: A family of hyperelastic models for human brain tissue. J. Mech. Phys. Solids 106, 60-79 (2017)

32. Mihai, L.A., Chin, L., Janmey, P.A., Goriely, A.: A comparison of hyperelastic constitutive models applicable to brain and fat tissues. J. R. Soc. Interface. 12, 20150486 (2015)

33. Mihai, L.A., Fitt, D., Woolley, T.E., Goriely, A.: Likely cavitation in stochastic elasticity. J. Elast. 137(1), 27-42 (2019). https://doi.org/ 10.1007/s10659-018-9706-1

34. Mihai, L.A., Fitt, D., Woolley, T.E., Goriely, A.: Likely equilibria of stochastic hyperelastic spherical shells and tubes. Math. Mech. Solids 24(7), 2066-2082 (2019). https://doi.org/10.1177/1081286518811881

35. Mihai, L.A., Fitt, D., Woolley, T.E., Goriely, A.: Likely oscillatory motions of stochastic hyperelastic solids. Trans. Math. Appl. 3, 1-42 (2019). https://doi.org/10.1093/imatrm/tnz003

36. Mihai, L.A., Goriely, A.: How to characterize a nonlinear elastic material? a review on nonlinear constitutive parameters in isotropic finite elasticity. Proc. R. Soc. A 473, 20170607 (2017). https://doi.org/10.1098/rspa.2017.0607

37. Mihai, L.A., Woolley, T.E., Goriely, A.: Stochastic isotropic hyperelastic materials: constitutive calibration and model selection. Proc. R. Soc. A 474, $20170858(2018)$

38. Mihai, L.A., Woolley, T.E., Goriely, A.: Likely equilibria of the stochastic Rivlin cube. Phil. Trans. R. Soc. A 377, 20180068 (2019). https://doi.org/10.1098/rsta.2018.0068

39. Mihai, L.A., Woolley, T.E., Goriely, A.: Likely chirality of stochastic anisotropic hyperelastic tubes. Int. J. Non-Linear Mech. 114, 9-20 (2019). https://doi.org/10.1016/j.ijnonlinmec.2019.04.004

40. Mihai, L.A., Woolley, T.E., Goriely, A.: Likely cavitation and radial motion of stochastic elastic spheres. Nonlinearity, under review. arXiv:1906.10514 (2019)

41. Misra, S., Ramesh, K.T., Okamura, A.M.: Modelling of non-linear elastic tissues for surgical simulation. Comput. Methods Biomech. Biomed. Eng. 13, 811-818 (2010)

42. Mooney, M.: A theory of large elastic deformation. J. Appl. Phys. 11, 582-592 (1940)

43. Mumford, D.Arnold, V., Atiyah, M., Lax, P., Mazur, B. (eds.): The dawning of the age of stochasticity, Mathematics: Frontiers and Perspectives. American Mathematical Society, Providence (2000)

44. Nörenberg, N., Mahnken, R.: Parameter identification for rubber materials with artificial spatially distributed data. Comput. Mech. 56, 353-370 (2015)

45. Oden, J.T.: Adaptive multiscale predictive modelling. Acta Numer. 27, 353-450 (2018)

46. Oden, J.T., Prudencio, E.E., Hawkins-Daarud, A.: Selection and assessment of phenomenological models of tumor growth. Math. Models Methods Appl. Sci. 23, 1309-1338 (2013)

47. Ogden, R.W.: Large deformation isotropic elasticity - on the correlation of theory and experiment for incompressible rubberlike solids. Proc. R. Soc. Lond. A 326, 565-584 (1972)

48. Ogden, R.W. Non-linear elastic deformations, 2nd edn. Dover, New York (1997)

49. Ogden, R.W., Saccomandi, G., Sgura, I.: Fitting hyperelastic models to experimental data. Comput. Mech. 34, 484-502 (2004)

50. Ostoja-Starzewski, M.: Microstructural randomness and scaling in mechanics of materials. CRC Press, Chapman and Hall (2007)

51. Pearson, K.: On the criterion that a given system of deviations from the probable in the case of a correlated system of variables is such that it can be reasonably supposed to have arisen from random sampling. Philos. Mag. Ser. 5 50(302), 157-175 (1900). https://doi.org/10.1080/14786440009463897

52. Pearson, E.S., Wishart, J.: "Student's" collected papers, issued by the biometrika office. University College, London (1942)

53. Pucci, E., Saccomandi, G.: A note on the Gent model for rubber-like materials. Rubber Chem. Technol. 75, 839-852 (2002)

54. Rivlin, R.S.: Large elastic deformations of isotropic materials. IV. Further developments of the general theory. Philos. Trans. R. Soc. Lond Ser. A Math. Phys. Sci. 241, 379-397 (1948)

55. Rivlin, R.S., Saunders, D.W.: Large elastic deformations of isotropic materials. VII. Experiments on the deformation of rubber. Philos. Trans. R. Soc. Lond. A 243(865), 251-288 (1951)

56. Robert, C.P. The Bayesian choice: from decision-theoretic foundations to computational implementation, 2nd edn. Springer, New York (2007)

57. Shannon, C.E.: A mathematical theory of communication. Bell Syst. Techn. J 27, 379-423,623-659 (1948)

58. Soize, C.: A nonparametric model of random uncertainties for reduced matrix models in structural dynamics. Probab. Eng. Mech. 15, 277-294 (2000)

59. Soize, C.: Maximum entropy approach for modeling random uncertainties in transient elastodynamics. J. Acoust. Soc. Amer. 109, 1979-1996 (2001)

60. Soize, C.: Non-gaussian positive-definite matrix-valued random fields for elliptic stochastic partial differential operators. Comput. Methods Appl. Mech. Eng. 195, 26-64 (2006)

61. Soize, C.: Stochastic modeling of uncertainties in computational structural dynamics - Recent theoretical advances. J. Sound Vib. 332, 2379-2395 (2013)

62. Soize, C.: Uncertainty quantification: an accelerated course with advanced applications in computational engineering interdisciplinary applied mathematics book, vol. 47. Springer, New York (2017)

63. Sommer, G., Eder, M., Kovacs, L., Pathak, H., Bonitz, L., Mueller, C., Regitnig, P., Holzapfel, G.A.: Multiaxial mechanical properties and constitutive modeling of human adipose tissue: a basis for preoperative simulations in plastic and reconstructive surgery. Acta Biomater. 9, 9036-9048 (2013)

64. Soni, J., Goodman, R.: A mind at play: how Claude Shannon invented the information age. Simon \& Schuster, New York (2017) 
65. Staber, B., Guilleminot, J.: Stochastic modeling of a class of stored energy functions for incompressible hyperelastic materials with uncertainties. Comptes Rendus Mécanique 343, 503-514 (2015)

66. Staber, B., Guilleminot, J.: Stochastic modeling of the Ogden class of stored energy functions for hyperelastic materials: the compressible case. J. Appl. Math. Mech./Z. Angewandte Math. Mech. 97, 273-295 (2016)

67. Staber, B., Guilleminot, J.: Stochastic hyperelastic constitutive laws and identification procedure for soft biological tissues with intrinsic variability. J. Mech. Behav. Biomed. Mater. 65, 743-752 (2017)

68. Staber, B., Guilleminot, J.: A random field model for anisotropic strain energy functions and its application for uncertainty quantification in vascular mechanics. Comput. Methods Appl. Mech. Eng. 333, 94-113 (2018)

69. Staber, B., Guilleminot, J., Soize, C., Michopoulos, J., Iliopoulos, A.: Stochastic modeling and identification of an hyperelastic constitutive model for laminated composites. Comput. Methods Appl. Mech. Eng. 347, 425-444 (2019)

70. Steinmann, P., Hossain, M., Possart, G.: Hyperelastic models for rubber-like materials: consistent tangent operators and suitability for Treloar's data. Arch. Appl. Mech. 82, 1183-1217 (2012)

71. Sullivan, T.J.: Introduction to uncertainty quantification. Springer, New York (2015)

72. Sutton, M., Orteu, J.J., Schreire, H.W.: Image correlation for shape, motion and deformation measurements: basic concepts theory and applications. Springer, New York (2009)

73. Thorburn, W.M.: The myth of Occam's razor. Mind 27, 345-353 (1918)

74. Titterington, D.M.: Irreverent Bayes. J. Appl. Stat. 9(1), 16-18 (1982). https://doi.org/10.1080/02664768200000003

75. Treloar, L.R.G. The physics of rubber elasticity, 3rd edn. Oxford University Press, Oxford, UK (2005)

76. Truesdell, C., Noll, W. The non-linear field theories of mechanics, 3rd edn. Springer, New York (2004)

77. Twizell, E.H., Ogden, R.W.: Non-linear optimization of the material constants in Ogden's stress-deformation function for incompressinle isotropic elastic materials. ANZIAM J. 24, 424-434 (1983)

78. Wyatt, H.L., Pullin, R., Yang, T.H.J., Evans, S.L.: Deformation during the electrosurgical vessel sealing process. Strain 52, 372-379 (2016)

79. Wyatt, H., Safar, A., Clarke, A., Evans, S.L., Mihai, L.A.: Nonlinear scaling effects in the stiffness of soft cellular structures. R. Soc. Open Sci. 6, 181361 (2019)

Publisher's note Springer Nature remains neutral with regard to jurisdictional claims in published maps and institutional affiliations. 